\title{
¿Ha llegado el momento de considerar la inclusión de la medicina del viajero en la formación del médico en América Latina?
}

\author{
Diego F. Cortés, Carlos E. Calvache, Angélica G. Badillo-Morales, Alfonso J. Rodríguez-Morales
}

La explosión actual del turismo, además de incontables beneficios, ha condicionado a los viajeros, tanto residentes de países de altos como de medios y bajos ingresos, a estar en contacto con diversos riesgos para la salud durante su estancia en diferentes destinos, tales como enfermedades infecciosas endémicas o lesiones asociadas a las actividades a realizar. Todo ello es factible e in crescendo dada la globalización y gran conectividad aérea en las diferentes regiones del mundo [1]. De ahí que es necesario implementar, aplicar y profundizar en un campo específico del conocimiento médico para controlar dichos problemas, como lo es la medicina del viajero o emporiatría, disciplina que ha emergido en las últimas dos décadas $[1,2]$.

$\mathrm{Su}$ aplicación ya se ha venido dando en las regiones más desarrolladas del mundo, entre ellas América del Norte y muchos países de Europa [2,3]. En los países de América Latina, la importancia dada a la prevención de enfermedades adquiridas durante un viaje había sido casi tan nula como el conocimiento del área encargada de tratarlas (obviando todos los 'pros' a los que se puede llegar con el simple hecho de prevenir la enfermedad de un viajero o el origen de un posible brote a partir de un caso importado que pueda generar transmisión en el lugar de residencia de un viajero retornante) [2-5]. Mientras que en países desarrollados ya se imparten maestrías, especializaciones y diplomaturas en medicina del viajero (por ejemplo, University of Otago, en Nueva Zelanda; Facultad de Medicina del Viajero, Royal College of Physicians and Surgeons of Glasgow, en Escocia, Reino Unido), en América Latina sólo se cuentan con algunos cursos (como el Curso Latinoamericano de Medicina del Viajero) $[1,3]$. Más aún, en la formación de pregrado, en las escuelas y programas de medicina no existen todavía cursos, asignaturas o materias regulares o elec- tivas que incluyan la formación concerniente a la aplicación de la emporiatría, dejando así la posibilidad de que problemas de salud del viajero de gran frecuencia epidemiológica no sean instruidos en personal que en un futuro deberá afrontarlos corrientemente, más aún dependiendo de los lugares donde ejerzan su práctica clínica $[3,6,7]$.

La inclusión de la medicina del viajero como área de enseñanza en pregrado traería consigo beneficios en los campos de su aplicación, mejorando además las condiciones de salud de los viajeros, dado que esta disciplina se orienta a la prevención de condiciones (consulta previaje) y su atención (durante y después del viaje) [5-7]. Por ello, la reflexión actual giraría en torno a si ha llegado el momento de considerar la inclusión de la medicina del viajero en la formación del médico en América Latina.

La gran mayoría de escuelas y programas de medicina de los diferentes países en la región deben pensar si requieren o no un ajuste en el cual se agreguen nuevas áreas relacionadas con la previsión de enfermedades asociadas a viajar, o al menos aumente la proporción de horas de cátedra de disciplinas relacionadas que puedan incluir contenidos específicos en medicina del viajero (como infectología, medicina tropical, salud pública, etc.). Es de esperar que en paralelo al aumento evidenciable en cuanto a logística, organización y servicios ofrecidos en los desplazamientos nacionales e internacionales, también se incrementen de forma suficientemente notable las medidas generales tomadas para prevenir y controlar enfermedades asociadas a viajes, con el fin de poder hacer frente a diferentes eventos epidemiológicos individuales y colectivos en los cuales se requiera una respuesta eficiente y adecuada en términos de acción y prevención médica. También sería de esperar una disminución de la prevalencia de enfermedades infecciosas como
Semillero de Investigación SIDA y Otras Enfermedades Infecciosas, Programa de Medicina (D.F. Cortés, C.E. Calvache, A.G. Badillo-Morales); Departamento de Medicina Comunitaria (A.J. RodríguezMorales); Facultad de Ciencias de la Salud; Universidad Tecnológica de Pereira. Sociedad Latinoamericana de Medicina del Viajero (A.J. Rodríguez-Morales). Pereira, Colombia.

Correspondencia: Dr. Alfonso J. Rodríguez Morales. Departamento de Medicina Comunitaria. Facultad de Ciencias de la Salud. Universidad Tecnológica de Pereira. Pereira 660001 Risaralda, Colombia.

E-mail: arodriguezm@utp.edu.co (c) $2014 \mathrm{FEM}$ 
dengue, malaria, fiebre amarilla, entre otras, particularmente en la población de viajeros.

La medicina del viajero es un área que, a pesar de ser nueva, genera muchos beneficios y no debería considerarse como secundaria en el perfil médico, por lo cual parece ser el momento de buscar las formas de incluirla en la enseñanza médica de pregrado en América Latina

\section{Bibliografía}

1. Hill DR, Ericsson CD, Pearson RD, Keystone JS, Freedman DO, Kozarsky PE, et al; Infectious Diseases Society of America. The practice of travel medicine: guidelines by the Infectious Diseases Society of America. Clin Infect Dis 2006; 43: 1499-539.
2. Rodríguez-Morales AJ, Palacios H. Trends in the publication of scientific research in travel medicine from Latin America. Travel Med Infect Dis 2009; 7: 323-4.

3. Rodríguez-Morales AJ, Zuckerman JN. Extending across continents: travel medicine and Latin America. Travel Med Infect Dis 2012; 10: 55-6.

4. Risquez A, Marrero A, Naranjo N, Palacios Y, Rossomando MT, Rodríguez-Morales AJ. Diseases and injuries associated with travel among students, employees and teachers of the Central University of Venezuela during the national summer vacations. Travel Med Infect Dis 2010; 8: 41-6.

5. Rodríguez-Morales AJ, Orduña T, Lloveras S, Jones ME. Research in travel medicine and emerging regions: the need for teaching how to publish. Travel Med Infect Dis 2013; 11: 201-2.

6. Zuckerman JN, Chiodini P. Creating the future for travel medicine. Travel Med Infect Dis 2010; 8: 67.

7. Zuckerman JN. Grass roots growing into travel medicine. Travel Med Infect Dis 2009; 7: 1 . 